\title{
Was reizt junge Frauen am One-Night-Stand?
}

\author{
Fast ein Drittel der Studentinnen im ersten Semester, die sich auf \\ ein sexuelles Kurzabenteuer mit einem Mann eingelassen haben, \\ sieht in dieser Begegnung keine Vorteile für sich.
}

Hier steht eine Anzeige.

Springer
_ Das erste Jahr von
Studenten an US-ame-
rikanischen Colleges
gilt als eine Zeit hefti-
ger sexueller Aktivität.
262 Erstsemester-Stu-
dentinnen im Durch-
schnittsalter von 18,1
Jahren wurden be-
fragt, welche Motive
sie bewegt hatten, eine
sexuelle Kurzzeit-Be-
ziehung ohne weitere
Pră

Präliminarien oder Absichten mit einem bislang weitgehend unbekannten Kommilitonen einzugehen. Zudem sollten sie darlegen, welche Vorteile sie aus dieser Begegnung für sich ziehen konnten.

Von den zehn in einem Fragebogen vorgegebenen Vorteilen wählten die Studentinnen am häufigsten, nämlich zu $23 \%$, pures sexuelles Vergnügen aus. 21\% gaben allgemeine positive Gefühle an, $11 \%$ fühlten sich als Person bestätigt, und $11 \%$ erlebten die Begegnung als wichtigen Beitrag zur Klärung ihrer Gefühle. Unter $10 \%$ gaben an, mit dem ersten zufälligen Sexualpartner eine dauerhafte freundschaftliche oder romantische Beziehung aufgebaut zu haben. Ebenfall unter $10 \%$ spürten eine stärkere Zugehörigkeit zu einer Gruppe.

Insgesamt gaben $71 \%$ der Studentinnen zumindest einen Vorteil an - aber immerhin 29\% konnten für sich aus dem Sexualkontakt überhaupt keinen Vorteil ersehen.

- Shepardson RL et al. Benefits of hooking up: self-reports from first year college women. Int J Sex Health. 2016;28:216-20

\section{KOMMENTAR}

Frühere Untersuchungen zu diesem Thema legten den Schwerpunkt vor allem auf die negativen Konsequenzen flüchtiger sexueller Beziehungen - insbesondere solche für Frauen. Dazu gehören Emotionen wie Reue und Enttäuschung, Scham, Vereinsamung, Verachtung und Depression sowie das Gefühl, ein Opfer zu sein. Und auch sexuell übertragbare Erkrankungen waren immer wieder Gegenstand der Betrachtung.

Diese neue Befragung fokussiert hingegen auf die Vorteile solcher sexueller „Abenteuer". Die mag es sicher geben, doch insgesamt sind die Daten eher ernüchternd. Aus vollen Rohren werden wir von Werbung und Medien mit der Botschaft bombardiert, dass Sex angeblich die schönste Sache der Welt sei. In der vorliegenden Untersuchung gab nicht einmal ein Viertel der Frauen an, dass der One-Night-Stand ihnen Spaß gemacht habe. Diese Daten stehen im Einklang mit anderen detaillierten $\mathrm{Be}$ fragungen, die zeigten, dass nur zwischen 11 und 34\% der Frauen bei flüchtigen sexuellen Begegnungen einen Orgasmus haben. Das medial vermittelte Bild und die Realität scheinen also doch recht weit auseinander zu klaffen.

Prof. Dr. med. H. S. FüeßI 